\title{
Valency configuration of transition metal impurities in $\mathrm{ZnO}$
}

\author{
L. Petit ${ }^{1}$, T. C. Schulthess ${ }^{1}$, A. Svane ${ }^{2}$, W.M. Temmerman ${ }^{3}$, Z. Szotek ${ }^{3}$, and A. Janotti ${ }^{4}$ \\ ${ }^{1}$ Computer Science and Mathematics Division, \\ and Center for Computational Sciences, \\ Oak Ridge National Laboratory, Oak Ridge, TN 37831, USA \\ 2 Department of Physics and Astronomy, \\ University of Aarhus, DK-8000 Aarhus C, Denmark \\ ${ }^{3}$ Daresbury Laboratory, Daresbury, Warrington WA4 4AD, UK \\ ${ }^{4}$ Materials Department, University of California, Santa Barbara, CA 93106-5050
}

(Dated: October 5, 2018)

\begin{abstract}
We use the self-interaction corrected local spin-density approximation to investigate the ground state valency configuration of transition metal $(\mathrm{TM}=\mathrm{Mn}, \mathrm{Co})$ impurities in n- and p-type $\mathrm{ZnO}$. We find that in pure $\mathrm{Zn}_{1-x} \mathrm{TM}_{x} \mathrm{O}$, the localized $\mathrm{TM}^{2+}$ configuration is energetically favoured over the itinerant $d$-electron configuration of the LSD picture. Our calculations indicate furthermore, that the $(+/ 0)$ donor level is situated in the $\mathrm{ZnO}$ gap. Consequently, for n-type conditions, with the Fermi energy $\epsilon_{F}$ close to the conduction band minimum, TM remains in the $2+$ charge state, whilst for p-type conditions, with $\epsilon_{F}$ close to the valence band maximum, the $3+$ charge state is energetically preferred. In the latter scenario, modelled here by co-doping with $\mathrm{N}$, the additional delocalized $d$-electron charge transfers into the hole states at the top of the valence band, and hole carriers will only exist, if the $\mathrm{N}$ concentration exceeds the TM impurity concentration.

PACS numbers:
\end{abstract}


Based on the possible interplay between electronic properties and spin-functionality, diluted magnetic semiconductors (DMS) are expected to play a major part in the development of the next generation of electronic devices. $\stackrel{1}{-}$ The considerable challenge consists in designing materials that remain ferromagnetic above room temperature. In Mn doped GaAs, where ferromagnetism is well established, the currently highest achieved Curie temperature is $\mathrm{T}_{C}$ $\sim 170 \mathrm{~K}$. Ferromagnetism at even higher temperatures has been reported in some semiconductors (for a review, see for example Pearton et $a l^{2}{ }^{2}$ ), but doubts persist as to the carrier induced nature of the observed magnetic order. Recently, the prediction by Dietl et $a l .{ }^{3}$ of room temperature ferromagnetism in p-type $\mathrm{Zn}_{1-x} \mathrm{Mn}_{x} \mathrm{O}$ has generated considerable research activity, both in theory and experiment. So far, no conclusive experimental evidence has emerged that could either confirm or disprove the prediction. Various experimental investigations of the magnetic order in $\mathrm{Zn}_{1-x} \mathrm{TM}_{x} \mathrm{O}$ give contradictory results, ranging from spin glass ${ }^{\underline{\underline{4}}}$ and antiferromagnetic behaviour ${ }^{5.6}$ to ferromagnetism below ${ }^{\underline{7}}$ or even above $e^{8.9 .10}$ room temperature. It has also been suggested that the observed ferromagnetism might be due to the formation of secondary manganese oxide phases. ${ }^{11}$ Generally, the observed magnetic properties of the DMS seem to depend strongly on the method by which they are synthesized. From all these conflicting results, the overall picture (though not universally accepted) seems to emerge, that $\mathrm{Zn}_{1-x} \mathrm{TM}_{x} \mathrm{O}$ will not be ferromagnetic, 12 unless additional hole or electron carriers are co-doped. Comprehensive overviews on the current state of research into $\mathrm{ZnO}$ spintronics can be found in references [13,14,15, 16].

From the theory point of view, there are currently two main approaches to modelling dilute magnetic semiconductors that differ rather fundamentally in the way the transition metal $d$-electrons are described. In the Zener model approach proposed by Dietl et $a l .^{3}$ it is assumed that the on-site $d$ - $d$ correlations are significant enough to prevent hopping, causing the transition metal $d$-electrons to remain localized on each their sites. In the band model approach it is assumed that the gain in kinetic energy associated with $d$-electron delocalization is large enough to overcome the on-site correlations, and that consequently the $d$-electrons fill up the resulting exchange split $d$-band, situated in the case of $\mathrm{ZnO}$ in the middle of the gap. Both the Zener model description, $\frac{3}{-}$ and the band-electron picture $\frac{17,18}{}$ have been put forward to predict high temperature (even room temperature) ferromagnetism in TM doped p-type $\mathrm{ZnO}$.

Despite this apparent agreement on ferromagnetism, the two models nevertheless differ 
with respect to the proposed mechanism, which ultimately can be traced back to the very different assumptions regarding the ground state valency configuration of the TM impurities. With the self-interaction corrected (SIC)-LSD approximation, used in the present work, $\stackrel{19.20}{ }$ delocalized and localized $d$-electrons are treated on an equal footing, by removing an unphysical self-interaction, inherent in LSD, for every localized d-electron ${ }^{21}$ Thus the localized mean field scenario, and the delocalized bandstructure scenario can be compared, and the ground state configuration of the TM-ion can be deduced from the global energy minimum.

\section{SIC-LSD METHOD}

Whereas density functional theory (DFT) is in principle exact, the very popular local spin density approximation (LSD) used to describe exchange and correlation introduces an unphysical self-interaction. This can be demonstrated on a single electron moving in an external potential, which should be described by the total energy functional

$$
E^{D F T}\left[n_{\alpha}\right]=E_{k i n}\left[n_{\alpha}\right]+V_{e x t}\left[n_{\alpha}\right]
$$

but which in the LSD approximation is given by

$$
E^{L S D}\left[n_{\alpha}\right]=E_{k i n}\left[n_{\alpha}\right]+V_{e x t}\left[n_{\alpha}\right]+U_{H}\left[n_{\alpha}\right]+E_{x c}^{L S D}\left[n_{\alpha}\right]
$$

Here, $\mathrm{U}_{H}\left[\mathrm{n}_{\alpha}\right]$ and $\mathrm{E}_{x c}^{L S D}\left[\mathrm{n}_{\alpha}\right]$ are respectively the self-Hartree and self-exchange-correlation energies, for the single electron density $\left[\mathrm{n}_{\alpha}\right]$, and together defining the self-interaction as

$$
\delta_{\alpha}^{S I C}=U_{H}\left[n_{\alpha}\right]+E_{x c}^{L S D}\left[n_{\alpha}\right]
$$

In the solid, using the SIC-LSD method, the self-interaction for each occupied orbital is subtracted from the LSD functional

$$
E^{S I C}\left[\left\{\psi_{\alpha}\right\}\right]=E^{L S D}[n]-\sum_{\alpha}^{o c c} \delta_{\alpha}^{S I C},
$$

where the total energy $E^{S I C}\left[\left\{\psi_{\alpha}\right\}\right]$ is expressed as functional of the set of orbitals $\psi_{\alpha}$, and $n$ is the total charge density of the system. For states of Bloch symmetry in an infinite crystal, i.e. band states, the self-interaction vanishes. To benefit from the self-interaction correction, a localized state must be formed. The ensuing many-electron wavefunction becomes a mixture 
of Bloch-type and Heitler-London type one particle states, i.e. delocalized and localized delectrons coexist. The delocalized $d$-states together with the remaining valence electrons continue to be treated in the LSD approximation, whilst those $d$-states that are treated as localized gain the self-interaction (localization) energy, but loose the corresponding band formation energy. TM configurations with varying numbers of localized $d$-states - in general different 'localization scenarios' - can be explored, and compared with respect to their total energies. Consequently, the preferred ground state configuration, as far as the number of localized $d$-electrons is concerned, can be determined from the global energy minimum.

Different configurations of localized and delocalized $d$-electrons will give different nominal valencies, which in the SIC-LSD are defined as: $N_{\text {val }}=Z-N_{\text {core }}-N_{S I C}$, where $Z$ is the atomic charge, $N_{\text {core }}$ is the number of core and semicore electrons, and $N_{S I C}$ is the number of self-interaction corrected $d$-electrons respectively. According to this definition, a divalent TM valency refers to the localized $d^{5}$ configuration of the $\mathrm{Mn}$ ion $\left(\mathrm{Mn}^{2+}\right)$, and the localized

$d^{7}$ Co ion $\left(\mathrm{Co}^{2+}\right)$ respectively. The trivalent configuration has one less $d$-electron localized, and thus refers to the localized $d^{4} \mathrm{Mn}$ ion $\left(\mathrm{Mn}^{3+}\right)$ and $d^{6} \mathrm{Co}$ ion $\left(\mathrm{Co}^{3+}\right)$. The LSD picture corresponds to the scenarios with all $d$-electrons delocalized, i.e. the $d^{0}$ configuration for the Mn ion $\left(\mathrm{Mn}^{7+}\right)$ and the Co ion $\left(\mathrm{Co}^{9+}\right)$.

\section{BAND PICTURE VERSUS LOCALIZED PICTURE}

We use the SIC-LSD method to study the validity of the two above mentioned electronic structure models of TM impurities in $\mathrm{ZnO}$, and to determine which of the two assumes the correct TM ground state configuration, based on total energy considerations. When doping $\mathrm{ZnO}$ with $\mathrm{Mn} / \mathrm{Co}$, the TM ions occupy the Zn sites without changing the wurtzite structure. ${ }^{22}$ In our calculations, $\mathrm{Zn}_{1-x} \mathrm{TM}_{x} \mathrm{O}$ is modelled, by substituting a single $\mathrm{Zn}$ in a $(2 \times 2 \times 2)$ supercell consisting of $16 \mathrm{ZnO}$ formula units, with a TM impurity. The SICLSD scheme is implemented within the all-electron tight-binding linear-muffin-tin orbitals (TB-LMTO) method, with the atomic sphere approximation (ASA). Spin-orbit coupling is fully included in the self-consistency cycles. Empty spheres are introduced on high symmetry interstitial sites. The electron charge density was sampled using $27 k$-points in the irreducible wedge of the Brillouin zone. All calculations use the experimental lattice parameter. The resulting densities of states (DOS) for the delocalized $d$-electron scenario (LSD picture), and 


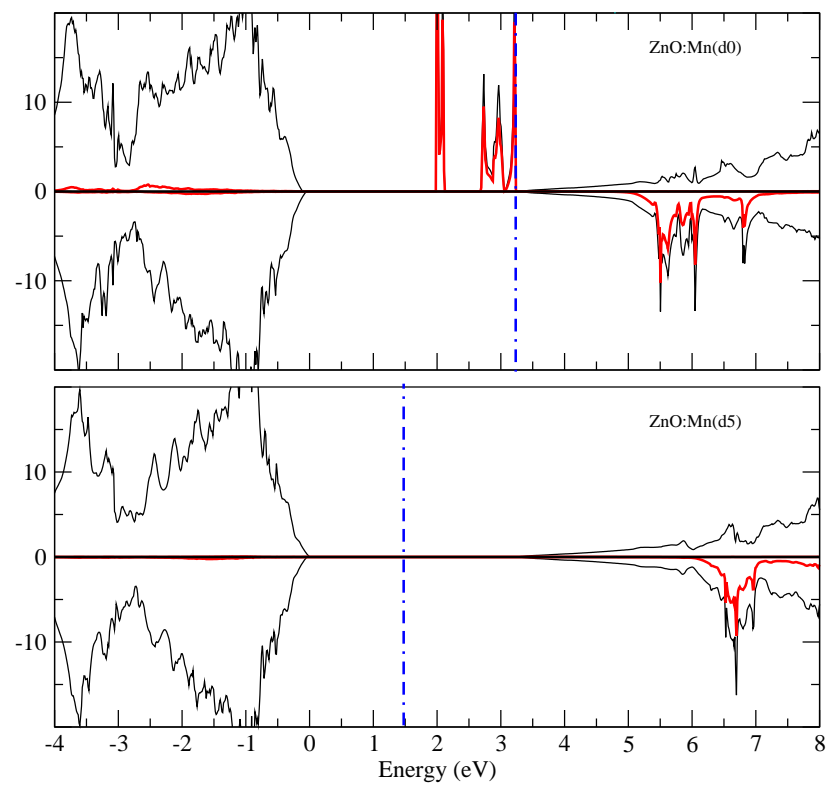

FIG. 1: Total (black), and Mn- $d$ projected (red) DOS as a function of energy, in states per eV, of $\mathrm{Zn}_{1-x} \mathrm{Mn}_{x} \mathrm{O}$ a) in the delocalized (band picture) $\mathrm{Mn}^{7+}$ configuration and b) in the localized divalent $\mathrm{Mn}^{2+}$ configuration.

the localized $d^{5}$ scenario are shown in Fig. 17 and Fig. 1b respectively.

In Fig. 1a, the delocalized electrons fully occupy the spin-up channel of the exchange split $d$-band whilst the spin-down band remains empty. The proponents of the band picture conclude that ferromagnetism, due to double-exchange, can not occur in this scenario. The same argument however is less useful for explaining the absence of ferromagnetism in $\mathrm{Zn}_{1-x} \mathrm{Co}_{x} \mathrm{O}$, where the two additional $d$-electrons start filling up the spin-down band, and the corresponding LSD DOS is reminiscent of a half-metal. In Fig. 1b, the $5 \mathrm{Mn} d$-electrons have been SI-corrected, and are therefore no longer available for band formation. Instead they form localized levels at energies below the valence band maximum (VBM). Note that the SIC-LSD, like any other density-functional theory, does not provide excited state properties. In particular, the approach cannot accurately determine the removal energies of localized states, and the bare SI-corrected d-bands always appear at too high binding energies due to the neglect of screening and relaxation effects, 23 and are therefore not shown in the DOS plots. The Fermi level is situated in the middle of the $\mathrm{ZnO}$ gap, and there are no free carriers, which for the proponents of the localized model explains the absence of carrier mediated ferromagnetism. The corresponding DOS for $\mathrm{Zn}_{1-x} \mathrm{Co}_{x} \mathrm{O}$ is quite similar to Fig. 
1b, but with seven Co $d$-states now SI-corrected in the localized scenario. Comparing the total energies for both models (columns 2 and 5 in Table I), we find that the localized $\mathrm{Mn}^{2+}$ and $\mathrm{Co}^{2+}$ scenarios are energetically more favourable than the corresponding band scenarios, by respectively $3 \mathrm{eV}$ for $\mathrm{Zn}_{1-x} \mathrm{Mn}_{x} \mathrm{O}$ (row 2) and $5.5 \mathrm{eV}$ for $\mathrm{Zn}_{1-x} \mathrm{Co}_{x} \mathrm{O}$ (row 8).

The band picture is clearly not preferred energetically, relative to the localized picture, however we still need to investigate whether the global energy minimum is obtained for an intermediate valency configuration of coexisting localized and delocalized $d$-electrons. In Fig. 2 the DOS of $\mathrm{Zn}_{1-x} \mathrm{Mn}_{x} \mathrm{O}$ is shown with the $\mathrm{Mn}$ ion in the $\mathrm{Mn}^{3+}$ configuration, i.e. only four of the $d$-electrons are localized by the SIC. The remaining $d$-electron, which is now treated in the LSD approximation, fills up the small $d$-peak situated in the middle of the $\mathrm{ZnO}$ gap. From Table I (row 2, columns 2 and 3), we see that this configuration is energetically less favourable than the $\mathrm{TM}^{2+}$ configuration. Similarly for Co doped $\mathrm{ZnO}$ the global energy minimum is obtained in the $\mathrm{Co}^{2+}$ scenario (Table I, row 8), and we conclude that both $\mathrm{Zn}_{1-x} \mathrm{Mn}_{x} \mathrm{O}$ and $\mathrm{Zn}_{1-x} \mathrm{Co}_{x} \mathrm{O}$ prefer the localized $d$-electron ground state configuration. The $\mathrm{TM}^{2+}$ configuration of transition metal impurities in $\mathrm{ZnO}$ is in agreement with experiment,, 24.25 and in support of the electronic structure underlying the Zener model, rather than the standard band picture.

TABLE I: Total energies in eV of Mn/Co doped p- and n-type $\mathrm{ZnO}$.

\begin{tabular}{|r|r|r|r|r|}
\hline \hline Compound & $\mathrm{Mn}^{2+}\left(d^{5}\right)$ & $\mathrm{Mn}^{3+}\left(d^{4}\right)$ & $\mathrm{Mn}^{4+}\left(d^{3}\right)$ & $\mathrm{Mn}^{7+}\left(d^{0}\right)$ \\
\hline $\mathrm{Zn}_{15 / 16} \mathrm{Mn}_{1 / 16} \mathrm{O}$ & $\mathbf{- 1 1 3 . 1 3}$ & -112.61 & -112.03 & -110.12 \\
\hline $\mathrm{Zn}_{15 / 16} \mathrm{Mn}_{1 / 16} \mathrm{O}_{15 / 16} \mathrm{~N}_{1 / 16}$ & -111.40 & $\mathbf{- 1 1 3 . 7 0}$ & -112.58 & \\
\hline $\mathrm{Zn}_{15 / 16} \mathrm{Mn}_{1 / 16} \mathrm{O}_{14 / 16} \mathrm{~N}_{2 / 16}$ & -110.80 & $\mathbf{- 1 1 1 . 4 8}$ & -111.35 & \\
\hline $\mathrm{Zn}_{14 / 16} \mathrm{Mn}_{1 / 16} \mathrm{Ga}_{1 / 16} \mathrm{O}$ & $\mathbf{- 1 1 5 . 9 1}$ & -115.40 & & \\
\hline $\mathrm{Zn}_{14 / 16} \mathrm{Mn}_{1 / 16} \mathrm{Sn}_{1 / 16} \mathrm{O}$ & $\mathbf{- 1 1 0 . 4 9}$ & -109.89 & & \\
\hline \hline & $\mathrm{Co}^{2+}\left(d^{7}\right)$ & $\mathrm{Co}^{3+}\left(d^{6}\right)$ & $\mathrm{Co}^{4+}\left(d^{5}\right)$ & $\mathrm{Co}^{9+}\left(d^{0}\right)$ \\
\hline $\mathrm{Zn}_{15 / 16} \mathrm{Co}_{1 / 16} \mathrm{O}$ & $\mathbf{- 1 1 4 . 0 4}$ & -113.40 & -112.70 & -108.38 \\
\hline $\mathrm{Zn}_{15 / 16} \mathrm{Co}_{1 / 16} \mathrm{O}_{15 / 16} \mathrm{~N}_{1 / 16}$ & -112.31 & $\mathbf{- 1 1 3 . 9 0}$ & -113.25 & \\
\hline $\mathrm{Zn}_{15 / 16} \mathrm{Co}_{1 / 16} \mathrm{O}_{14 / 16} \mathrm{~N}_{2 / 16}$ & -110.28 & $\mathbf{- 1 1 2 . 0 1}$ & -111.79 & \\
\hline $\mathrm{Zn}_{14 / 16} \mathrm{Co}_{1 / 16} \mathrm{Sn}_{1 / 16} \mathrm{O}$ & $\mathbf{- 1 1 1 . 4 8}$ & -110.89 & & \\
\hline \hline \hline
\end{tabular}




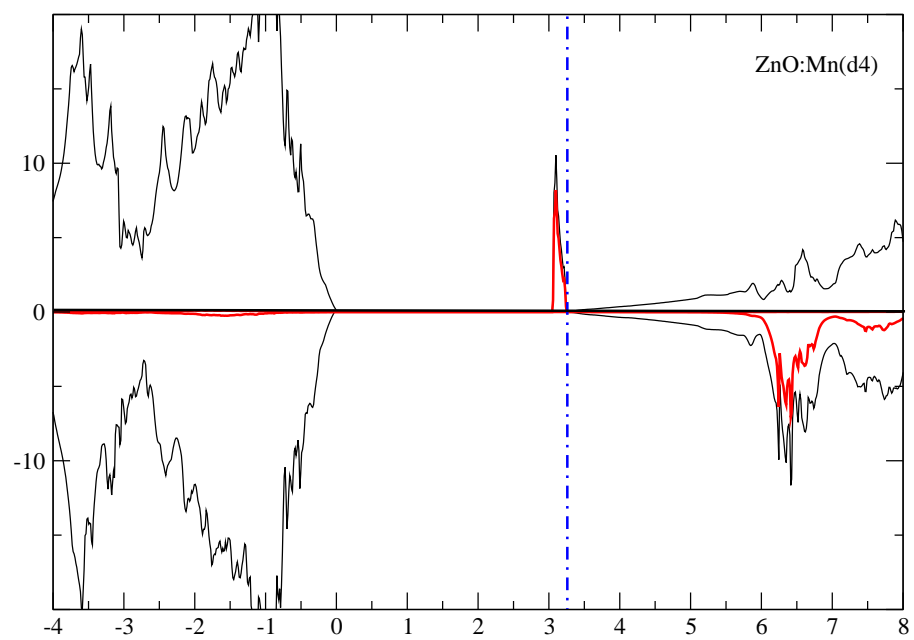

FIG. 2: Total (black), and Mn- $d$ projected (red) DOS, in states per eV, as a function of energy, in $\mathrm{eV}$, of $\mathrm{Zn}_{1-x} \mathrm{Mn}_{x} \mathrm{O}$ in the trivalent $\mathrm{Mn}^{3+}$ configuration.

In Fig. 2. the gap state that forms when treating an additional $d$-electron as band electron, is relatively narrow, and completely filled. The corresponding gain in band formation energy is insufficient to overcome the loss in SI-energy, and the $d$-electron prefers to remain localized. If however the Fermi level could be positioned below this gap state, for example through additional acceptor dopants, one could very well imagine that the resulting gain in energy from charge transfer to the Fermi level would be large enough for the delocalization to occur. The dependence on Fermi level position can be expressed through the formation energy

$$
E_{f}\left(Z n O: T M^{v}, q\right)=E_{t o t}\left(Z n O: T M^{v}, q\right)-E_{t o t}(Z n O)-\mu_{Z n}+\mu_{T M}+q \epsilon_{F}
$$

where $\left(\mathrm{ZnO}: \mathrm{TM}^{v}, \mathrm{q}\right)$ refers to an impurity of valency $v$, and charge $q, \mu_{Z n}$ and $\mu_{T M}$ are the respective chemical potentials, $\epsilon_{F}$ is the Fermi energy, and $\mathrm{E}_{t o t}$ the total energy of the system with impurity. Three scenarios need to be considered: In the neutral charge states, the substitutional impurity can either assume the divalent configuration, $\mathrm{TM}^{2+} \equiv\left(\mathrm{ZnO}: \mathrm{TM}^{2+}, 0\right)$, which does not introduce states in the gap (Fig. 1), or the trivalent configuration $\mathrm{TM}^{3+} \equiv$ $\left(\mathrm{ZnO}: \mathrm{TM}^{3+}, 0\right)$ which has an electron $d$-state in the gap (Fig. 2). In the charged scenario $\left(\mathrm{ZnO}: \mathrm{TM}^{3+},+\right)$ the delocalized $d$-electron has been transferred to the conduction or acceptor band, ionizing the TM impurity into the positive charge state. The formation energy as a 


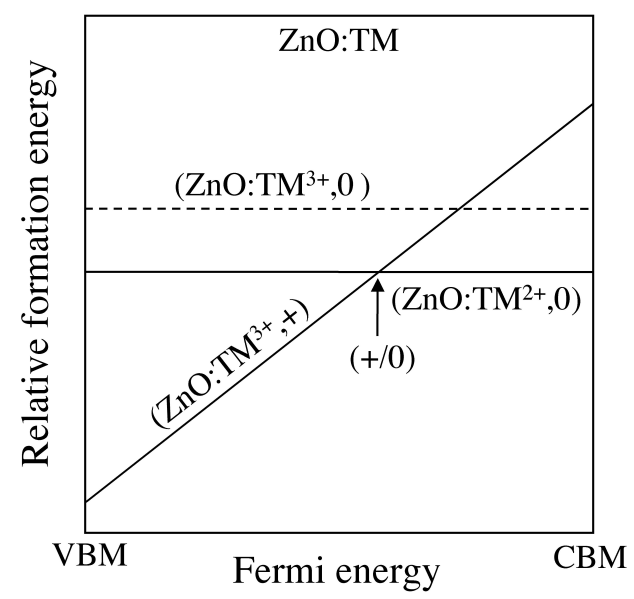

FIG. 3: Schematic plot of the formation energy as a function of Fermi energy for ZnO:TM, illustrating the link between the localization and delocalization picture and the TM donor ionization level in the band-gap. Only the $(+/ 0)$ level between the positively charged $\mathrm{TM}^{3+}$ and the neutral $\mathrm{TM}^{2+}$ valence configurations is indicated.

function of Fermi level is plotted in Fig. 3. The two horizontal lines correspond to the neutral scenarios, that are independent of the Fermi level position. The formation energy for the charged scenario changes linearly with Fermi level, as follows from equation (5), and as indicated by the skew line. The delocalization process

$$
T M^{2+} \rightarrow T M^{3+}+\text { electron }_{\epsilon_{F}}
$$

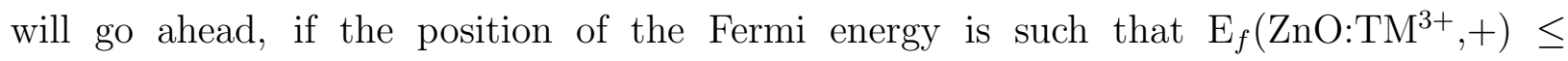
$\mathrm{E}_{f}\left(\mathrm{ZnO}: \mathrm{TM}^{2+}, 0\right)$. The donor transition level $(+/ 0)$ is defined by the Fermi energy above which the TM impurity is in the divalent state, and below which it is in the positively charged trivalent state. From SIC-LSD calculations, we have determined that the donor level in $\mathrm{Zn}_{1-x} \mathrm{TM}_{x} \mathrm{O}$ is positioned more or less centrally in the $\mathrm{ZnO}$ gap $\underline{26}$. The same conclusion can be drawn qualitatively from applying the numbers of Table I to Fig. 3: Since the neutral trivalent state $\left(\mathrm{ZnO}: \mathrm{TM}^{3+}, 0\right)$ has a gap state in the high energy part of the band gap, and since it is situated only $\sim 0.5 \mathrm{eV}$ above the neutral divalent $\left(\mathrm{ZnO}_{\mathrm{TM}}{ }^{2+}, 0\right)$ configuration, the donor level $(+/ 0)$ will be situated in the $3.4 \mathrm{eV}$ wide $\mathrm{ZnO}$ gap, as is also found experimentally: ${ }^{27}$

To investigate the actual effect of additional dopants on the electronic structure of $\mathrm{Zn}_{1-x} \mathrm{TM}_{x} \mathrm{O}$, we use the SIC-LSD to determine the ground state configuration for both 


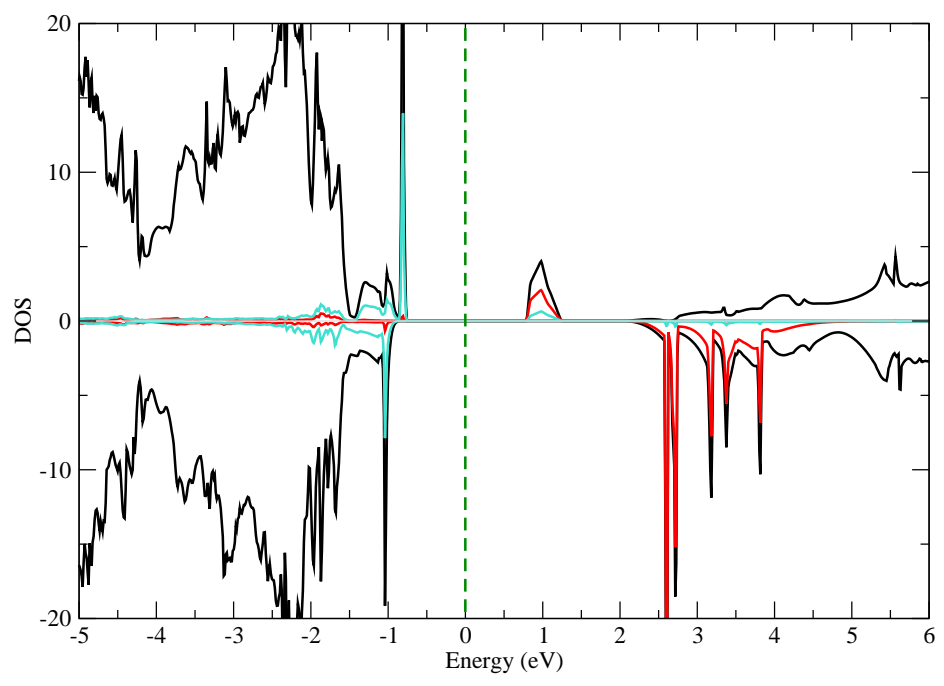

FIG. 4: Total (black), N-p(blue), and Mn- $d($ red) DOS as a function of energy, in states per eV, of $\mathrm{Zn}_{15 / 16} \mathrm{Mn}_{1 / 16} \mathrm{O}_{15 / 16} \mathrm{~N}_{1 / 16}$ in the trivalent $\mathrm{Mn}^{3+}$ ground state configuration.

p- and n-type scenarios. Acceptor (p-type) doping is achieved by replacing a single $\mathrm{O}$ in the supercell by $\mathrm{N}$, whereas n-type doping is modelled by replacing a single $\mathrm{Zn}$ atom by either Ga or Sn. The total energy results for the different dopants and different TM valency configurations are given in Table I. We find that, with $\mathrm{N}$ as additional dopant, the trivalent TM configuration becomes energetically most favourable, both for Mn (row 3) and Co (row 9). Nitrogen, having one p-electron less than O, acts as acceptor when introduced into $\mathrm{ZnO}$, with the corresponding acceptor level situated near the top of the valence band (Fig. 4). Given that the $(+/ 0)$ level is situated above the acceptor level, the delocalization process (6) can go ahead, leaving behind an empty $d$-band (gap state). The energy gain from charge transfer and hybridization in the $\mathrm{TM}^{3+}$ configuration is obviously large enough to overcome the corresponding loss in SIC (localization) energy.

In their paper on the possibility of magnetism in $\mathrm{Mn}$ doped $\mathrm{ZnO}$, Dietl et $a l \cdot \underline{\underline{3}}$ state that with n-type doping one can not expect ferromagnetic order, except maybe for very low temperature, but they predict room temperature ferromagnetism in the p-type scenario, where the hole carriers introduced by co-doping with $\mathrm{N}$ will mediate the interaction. The assumption is that the ground state configuration remains $\mathrm{Mn}^{2+}$, based on the argumentation that the $(+/ 0)$ level is situated below the $\mathrm{VBM}^{28}$ This picture is thus in contrast 
to our calculation, which finds the $(+/ 0)$ level situated above the $\mathrm{N}$ based acceptor states, and results in the $\mathrm{Mn}^{3+}$ ground state configuration. The importance of determining the correct ground state configuration becomes clear from Fig. 4, where the actual trivalent configuration implies charge transfer into the acceptor states, and absence of hole carriers, while a hypothetical divalent scenario with five localized $d$-states would imply absence of the gap state and charge transfer, leaving the $\mathrm{N}$ acceptor states partially unfilled (Dietl's scenario). From the SIC-LSD calculations it follows that, the band description of the $d$ states does not fully account for the correct electronic structure of the $\mathrm{Zn}_{1-x} \mathrm{TM}_{x} \mathrm{O}$ ground state, even when co-doped with N. But also the Zener model, with hole carriers mediating the magnetic interaction between localized spins residing on $\mathrm{TM}^{2+}$ ions, is not a true representation of the ground state. There are no hole carriers in either $\mathrm{Zn}_{15 / 16} \mathrm{Mn}_{1 / 16} \mathrm{O}_{15 / 16} \mathrm{~N}_{1 / 16}$ or $\mathrm{Zn}_{15 / 16} \mathrm{Co}_{1 / 16} \mathrm{O}_{15 / 16} \mathrm{~N}_{1 / 16}$ and carrier mediated ferromagnetism can therefore not occur.

It seems plausible that increasing the concentrations of the dopants, $[\mathrm{TM}]$ and $[\mathrm{N}]$, will not change the overall picture of fully compensated acceptor states, as long as $[\mathrm{TM}]=[\mathrm{N}]$. However, the qualitative picture changes considerably if we increase the relative amount of $\mathrm{N}$ impurities, i.e., if $[\mathrm{N}]>[\mathrm{TM}]$. Substituting two of the $\mathrm{O}$ atoms by $\mathrm{N}$, but with a single TM impurity in the 32 atom $\mathrm{ZnO}$ supercell, we find that, contrary to what one might expect, overdoping with $\mathrm{N}$ does not result in a further delocalization, i.e., a transition from $\mathrm{TM}^{3+}$ $\longrightarrow \mathrm{TM}^{4+}+\mathrm{e}^{-}$. From Table I we see that the $\mathrm{TM}^{3+}$ configuration gives the lowest total energy, both for $\mathrm{Zn}_{15 / 16} \mathrm{Mn}_{1 / 16} \mathrm{O}_{14 / 16} \mathrm{~N}_{2 / 16}$ (row 4), and $\mathrm{Zn}_{15 / 16} \mathrm{Co}_{1 / 16} \mathrm{O}_{14 / 16} \mathrm{~N}_{2 / 16}$ (row 10). The additional $\mathrm{N}$ impurity is thus not compensated by TM $d$-electrons which results in an impurity band at the top of the valence band, which is now only partially filled.

Co-doping with $\mathrm{N}$ has revealed itself to be rather difficult, ${ }^{29}$ which according to calculations by Yamamoto et al. ${ }^{30}$ can be related to the corresponding increase in the Madelung energy. Co-doping with electron donors, on the other hand, can be readily achieved. The theoretical prediction, that high temperature ferromagnetism will not occur in n-type Mndoped $\mathrm{ZnO}$, seems to be confirmed by experiment with respectively $\mathrm{Al}$ and $\mathrm{Ga}$ as co-dopants, however ferromagnetism has been reported in $\mathrm{Zn}_{1-x} \mathrm{Mn}_{x} \mathrm{O}$, when co-doped with $\mathrm{Sn}{ }^{31} \mathrm{In}$ the case of n-type doping, the substitution of Zn by either Ga or Sn introduces respectively one or two additional electrons into $\mathrm{ZnO}$, which results in an impurity band situated below the conduction band minimum (Fig. 5). From Table I, rows 5, 6, and 11, we see that in this case the divalent TM configuration remains energetically more favourable, i.e. given that 

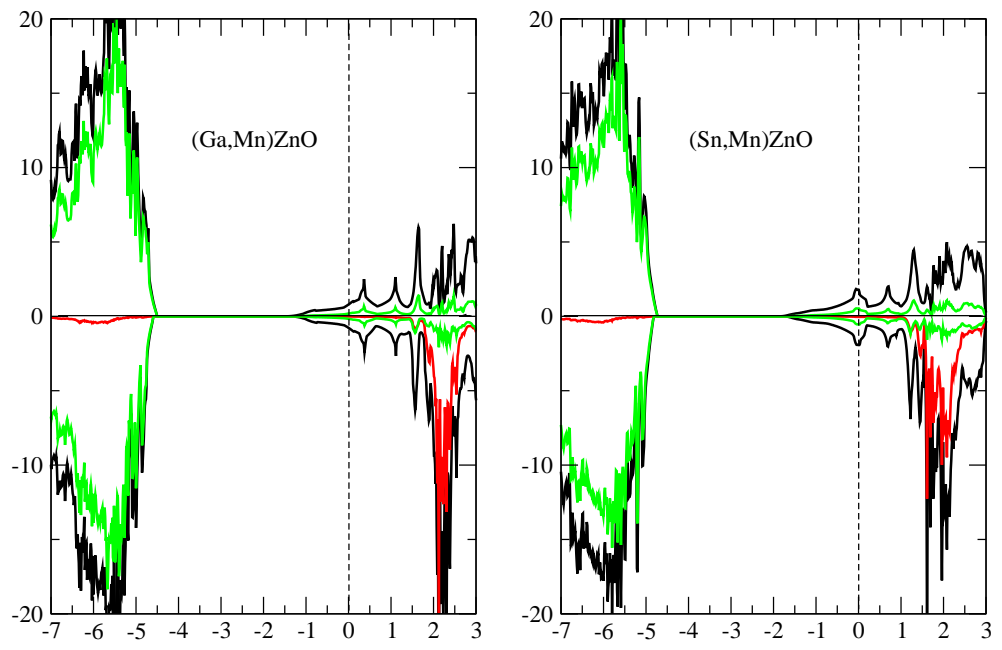

FIG. 5: Total DOS, in states per eV, as a function of energy, in eV, of a) $\mathrm{Zn}_{14 / 16} \mathrm{Mn}_{1 / 16} \mathrm{Ga}_{1 / 16} \mathrm{O}$ and b) $\mathrm{Zn}_{14 / 16} \mathrm{Mn}_{1 / 16} \mathrm{Sn}_{1 / 16} \mathrm{O}$, in the divalent $\mathrm{Mn}^{2+}$ configuration. The green, red, and black lines respectively represent the O- $p$ projected, Mn- $d$ projected, and total DOS's. The Fermi level (zero energy) is now situated in the conduction bands.

the Fermi level is here situated above the $(+/ 0)$ level, delocalization does not occur, and respectively five $(\mathrm{Mn})$ or seven (Co) $d$-electrons remain localized at each of the respective sites. With or without n-type co-dopant the divalent state (of both Mn and Co) invariably is 0.5-0.6 eV more favorable than the trivalent one, i.e. the n-type doping does not influence the TM configuration at all.

With respect to the calculated magnetic moments of the TM impurities in the various scenarios and valency configurations, we find that the orbital moment remains largely quenched. With no additional co-dopants, i.e. rows 2 and 7 of table I, the spin moments are approximately $4.5 \mu_{B}$ and $2.7 \mu_{B}$ for $\mathrm{Mn}$ and Co impurities respectively. These moments remain virtually unchanged regardless of the considered configuration, indicating for example that the SIC localized $d$-state of the $\mathrm{Mn}^{2+}$ configuration has the same moment as the filled narrow $d$-band state of the corresponding $\mathrm{Mn}^{3+}$ configuration. This situation changes when $\mathrm{N}$ is codoped (rows 3 and 9 of Table I). Here in in the $\mathrm{TM}^{2+}$ scenario, the moments are respectively $4.35 \mu_{B}$ for $\mathrm{Mn}$ in $\mathrm{Zn}_{15 / 16} \mathrm{Mn}_{1 / 16} \mathrm{O}_{1 / 16} \mathrm{~N}_{1 / 16}$ and $2.53 \mu_{B}$ for Co in $\mathrm{Zn}_{15 / 16} \mathrm{Co}_{1 / 16} \mathrm{O}_{1 / 16} \mathrm{~N}_{1 / 16}$. However for the corresponding $\mathrm{TM}^{3+}$ configuration, a $d$-electron has charge transfered into the hole state, with the moment being determined by the remaining SIC localized d-states, i.e. $\operatorname{Mn}\left(d^{4}\right)$ and $\operatorname{Co}\left(d^{6}\right)$. As a result, the magnetic moment of 
Mn decreases to $3.7 \mu_{B}$, and the magnetic moment of Co increases to $3.0 \mu_{B}$.

\section{CONCLUSION}

In summary, we have studied the electronic structure and different valency configurations of Co and Mn impurities in p- and n-type ZnO using the SIC-LSD ab initio method. From total energy considerations we find that the TM $d$-states remain localized if no additional hole donors are present. The $\mathrm{TM}^{2+}$ remains the ground state configuration under n-type conditions. The $\mathrm{TM}^{3+}$ becomes more favourable in $p$-type $\mathrm{ZnO}$, and the holes required for carrier mediated ferromagnetism occur only when the $\mathrm{N}$ co-dopant concentration exceeds the concentration of the TM impurities.

\section{Acknowledgments}

This work was supported in part by the Defense Advanced Research Project Agency and by the Division of Materials Science and Engineering, US Department of Energy. Oak Ridge National Laboratory is managed by UT-Batelle, LLC, for the US Department of Energy under Contract No. DE-AC05-00OR22725. The calculations were carried out at the Center for Computational Sciences at Oak Ridge National Laboratory, and at the Danish Center for Scientific Computing.

1 S. A. Wolf, D. D. Awschalom, R. A. Buhrman, J. M. Daughton, S. von Molnaŕ, M. L. Roukes, A. Y. Chtchelkanova, and D. M. Treger, Science 294, 1488 (2001).

2 S. J. Pearton, C. R. Abernathy, M. E. Overberg, G. T. Thaler, D. P. Norton, N. Theodorpoulou, A. F. Hebard, Y. D. Park, F. Ren, J. Kim, and L. A. Boatner, Appl. Phys. Lett. 93, 1 (2003).

3 T. Dietl, H. Ohno, F. Matsukura, J. Cibert, and D. Ferrand, Science 287, 1019 (2000).

4 T. Fukumura, Zhengwu Jin, M. Kawasaki, T. Shono, T. Hasegawa, S. Koshihara, and H. Koinuma, Appl. Phys. Lett. 78, 958 (2001).

5 A. S. Risbud, N. A. Spaldin, Z. Q. Chen, S. Stemmer, and Ram Seshadri, Phys. Rev. B 68, $205202(2003)$ 
6 S. W. Yoon, S. -B. Cho, S. C. We, S. Yoon, B. J. Suh, H. K. Song, and Y. J. Shin, J. Appl. Phys. 93, 7879 (2003).

7 S. W. Jung, S. -J. An, Gyu-Chul Yi, C. U. Jung, Sung-Ik Lee, and Sunglae Cho, Appl. Phys. Lett. 80, 4561 (2002).

8 P. Sharma, A. Gupta, K. V. Rao, F. J. Owens, R. Sharma, R. Ahuja, J. M. O. Guillen, B. Johansson, and G. A. Gehring,Nature materials 2, 673 (2003).

9 H. -T. Lin, T. -S. Chin, J. -C. Shih, S. -H. Lin, T. -M. Hong, R. -T. huang, F. -R. Chen, and J. -J. Kai, Appl. Phys. Lett. 85, 621 (2004).

10 M. H. F. Sluiter, Y. Kawazoe, P. Sharma, A. Inoue, A. R. Raju, C. Rout, and U. V. Waghmare, Phys. Rev. Lett. 94, 187204 (2005).

11 Y. M. Kim, M. Yoon, I. -W. Park, Y. J. Park, and Jong H. Lyou, Solid State Commun. 129, $175(2004)$.

12 G. Lawes, A. P. Ramirez, A. S. Risbud, and Ram Seshadri, cond-mat/0403196v1 (2004)

13 T. Fukumura, H. Toyasaki, and Y. Yamada, Semicond. Sci. Technol. 20, S103 (2005).

14 T. Fukumura, Y. Yamada, H. Toyasaki, T. Hasegawa, H. Koinuma, and M. Kawasaki, Appl. Surface Sci. 223, 62 (2004).

15 S. J. Pearton, W. H. Heo, M. Ivill, D. P. Norton, and T. Steiner, Semicond. Sci. Technol. 19, R59 (2004).

16 R. Janisch, P. Gopal, and N. A. Spaldin, J. Phys.: Condens. Matter 17, R657 (2005).

17 K. Sato, and H. Katayama-Yoshida, Semicond. Sci. Technol. 17, 367 (2002).

18 N. A. Spaldin, Phys. Rev. B 69, 125201 (2004).

19 A. Svane, Phys. Rev. B 53, 4275 (1996).

20 W. M. Temmerman, A. Svane, Z. Szotek and H. Winter, in Electronic Density Functional Theory: Recent Progress and New Directions, eited by J. F. Dobson, G. Vignale and M. P. Das (Plenum, New York, 1998), p. 327.

21 A. Zunger, J. P.Perdew, and G. L.Oliver, Solid State Commun. 34, 933 (1980),J. P. Perdew and A. Zunger, Phys. Rev. B 23, 5048 (1981).

22 T. Fukumura, Zhengwu Jin, A. Ohtomo, H. Koinuma, and M. Kawasaki, Appl. Phys. Lett. 75, 3366 (1999).

23 W. M. Temmerman, Z. Szotek, and H. Winter, Phys. Rev. B 47, 1184 (1993).

24 P. B. Dorain, Phys. Rev. 112, 1058 (1985). 
25 S. C. Wi et al., phys. stat. sol. (b) 241, 1529 (2004).

26 L. Petit, T. C. Schulthess, A. Svane, Z. Szotek, W. M. Temmerman, and A. Janotti, submitted.

27 O. Madelung, Semiconductors: Data Handbook, (Springer-Verlag, Berlin, 2004); D. A. Bonnell et al., J. Vac. Sci. Technol. B 9(2), 551 (1991).

28 T. Dietl, Semicond. Sci. Technol. 17, 377 (2002).

29 M. Joseph, H. Tabata, and T. Kawai, Jpn. J. Appl. Phys. 38, L1205 (1999).

30 T. Yamamoto, and H. Katayama-Yoshida, Jpn. J. Appl. Phys. 38, L166 (1998).

31 D. P. Norton, S. J. Pearton, A. F. Hebard, N. Theodoropoulo, L. A. Boatner, R. G. Wilson, Appl. Phys. Lett 82, 239 (2003). 